\title{
Complete hyperbolic Stein manifolds with prescribed automorphism groups
}

\author{
Su-Jen Kan
}

\begin{abstract}
It is well known that the automorphism group of a hyperbolic manifold is a Lie group. Conversely, it is interesting to see whether or not any Lie group can be prescribed as the automorphism group of a certain complex manifold.

When the Lie group $G$ is compact and connected, this problem has been completely solved by Bedford-Dadok and independently by Saerens-Zame in 1987. They have constructed strictly pseudoconvex bounded domains $\Omega$ such that $\operatorname{Aut}(\Omega)=G$. For Bedford-Dadok's $\Omega$, $0 \leq \operatorname{dim}_{\mathbb{C}} \Omega-\operatorname{dim}_{\mathbb{R}} G \leq 1$; for generic Saerens-Zame's $\Omega, \operatorname{dim}_{\mathbb{C}} \Omega \gg \operatorname{dim}_{\mathbb{R}} G$.

J. Winkelmann has answered affirmatively to noncompact connected Lie groups in recent years. He showed there exist Stein complete hyperbolic manifolds $\Omega$ such that $\operatorname{Aut}(\Omega)=G$. In his construction, it is typical that $\operatorname{dim}_{\mathbb{C}} \Omega \gg \operatorname{dim}_{\mathbb{R}} G$.

In this article, we tackle this problem from a different aspect. We prove that for any connected Lie group $G$ (compact or noncompact), there exist complete hyperbolic Stein manifolds $\Omega$ such that $\operatorname{Aut}(\Omega)=G$ with $\operatorname{dim}_{\mathbb{C}} \Omega=\operatorname{dim}_{\mathbb{R}} G$. Working on a natural complexification of the real-analytic manifold $G$, our construction of $\Omega$ is geometrically concrete and elementary in nature.
\end{abstract}

Mathematics Subject Classification (2000). 32C09, 32Q28, 32Q45, 53C24, 58D19.

Keywords. Hyperbolic manifolds, Stein manifolds, automorphism groups.

\section{Introduction}

It is well known that the automorphism group of a hyperbolic manifold has the structure of a Lie group. It is natural to ask whether every Lie group can appear as the automorphism group of certain hyperbolic manifolds or not. This problem sometimes is called the realization problem: realizing a Lie group as the automorphism group of a certain complex manifold.

This realization problem has been completely solved by Bedford-Dadok [B-D] and independently by Saerens-Zame [S-Z] when the given Lie group is compact and connected. In recent years, generalizing ideas of Saerens-Zame, Winkelmann [W] has settled the realizing problem for any connected Lie group. However, domains $\Omega$ constructed from this Saerens-Zame-Winkelmann's approach are typically with $\operatorname{dim}_{\mathbb{C}} \Omega \gg \operatorname{dim}_{\mathbb{R}} G$. 
The strategy of Saerens-Zame-Winkelmann was first to find a domain $D$ on which $G$ acts by automorphisms, and then perturbed it to a $G$-invariant strictly pseudoconvex subdomain in such a way that the additional automorphisms were ruled out by assigning CR-invariants to each $G$-orbit on the boundary. To find such a domain $D$ to start with, Saerens-Zame first embedded the compact Lie group $G$ into the unitary group $\mathrm{U}\left(N_{1}\right)$ and then constructed a domain $D$ in $\mathrm{GL}\left(N_{1}, \mathbb{C}\right) \times \mathbb{C}^{N_{2}}$ on which $G$ acts by automorphisms where $N_{1}$ and $N_{2}$ are large in general. Due to the above embedding process, the generic resultant complex manifold $\Omega$ has large complex dimension, $\operatorname{dim}_{\mathbb{C}} \Omega \gg \operatorname{dim}_{\mathbb{R}} G$. Having observed every Lie algebra is linear and hence the universal covering of a Lie group can be viewed as linear, Winkelmann has been able to find a group homomorphism $\tilde{G} \rightarrow \operatorname{Sp}\left(N_{3}, \mathbb{R}\right)$ and then find a suitable domain $D \subset \mathbb{C}^{N_{4}}$ to start with. Again, this embedding process and the construction of the domain $D$ has enormously increased the dimension.

The complexification $G_{\mathbb{C}}$ of a compact Lie group $G$ is Stein with $\operatorname{dim}_{\mathbb{C}} G_{\mathbb{C}}=$ $\operatorname{dim}_{\mathbb{R}} G$. Starting from domains in $G_{\mathbb{C}}$, Bedford-Dadok were able to give a more concrete construction. They found bounded strictly pseudoconvex domains $\Omega \subset G_{\mathbb{C}}$ or $\Omega \subset G_{\mathbb{C}} \times \mathbb{C}$ such that $\operatorname{Aut}(\Omega)=G$.

A natural attempt is to generalize Bedford-Dadok's approach to noncompact Lie groups. Unfortunately, it is not easy to give a canonical complexification of a generic noncompact Lie group such that $\operatorname{dim}_{\mathbb{C}} G_{\mathbb{C}}=\operatorname{dim}_{\mathbb{R}} G$. In this article we consider a special kind of complexifications of real-analytic manifolds to resolve this difficulty.

A Lie group $G$ equipped with a left-invariant metric $g$ is naturally a homogeneous space with $\operatorname{Isom}(G) \simeq L(G) \cdot K$ where $K$ is the isotropy group and $L(G)$ are left multiplications. We provide the tangent bundle of the Riemannian manifold $(G, g)$ with a complex structure in such a way that all the leaves of the Riemannian foliation are holomorphic curves. The disk bundle of radius $r$ is called a Grauert tube $T^{r} G$.

Generalizing the rigidity result, $\operatorname{Aut}\left(T^{r} G\right)=\operatorname{Isom}(G)$, proved in [Ka] we are able to dominate automorphism groups of certain domains $D \subset T^{r} G$. We prove it at Theorem 3.6 and call it the subrigidity $\operatorname{Aut}(D)<\operatorname{Isom}(G)$.

Starting from such a domain $D$ our strategy is to destroy, through certain $G$ invariant perturbations, the additional automorphisms coming from the isotropy group. Though the objects we deal with are not even relatively compact, the holomorphic extension property needed here is not hard to handle due to the special complex structure adopted here. In fact, most mappings we deal with are bundle mappings which automatically extend over the boundary. The main result of this article is

Theorem. Let $G$ be a connected Lie group of dimension $n \geq 2$. Then there exist complete hyperbolic Stein manifolds $\Omega, \operatorname{dim}_{\mathbb{C}} \Omega=n$, such that $\operatorname{Aut}(\Omega)=G$.

The dimensional condition $n \geq 2$ has to be added here since the main idea we shall use follows from the rigidity arguments of Grauert tubes while Grauert tubes 
and their perturbations are biholomorphic to the unit disc, by the Riemann mapping theorem, when the center is of dimension one. In the rigidity arguments of Grauert tubes, it is also necessary to assume the Riemannian manifold is connected. At this moment, I can not see a way to release the connectedness of the Lie groups. We remark while Saerens-Zame's result works for compact disconnected Lie group as well, the resultant complex manifold $\Omega$ is typically with $\operatorname{dim}_{\mathbb{C}} \Omega \gg \operatorname{dim}_{\mathbb{R}} G$.

The organization of this article is the following. In $\$ 2$ we briefly review terminologies concerning Grauert tubes and recall the existence of Stein Grauert tubes. Generalizing notations about the rigidity of Grauert tubes, we prove subrigidity characterization of certain domains in $\S 3$. In $\S 4$, specific domains and perturbations are constructed explicitly. We perturb domains in the tangent bundle of a connected Lie group in an invariant way such that extra symmetry on each fiber would be eliminated. By constructed such kind of domains in a fairly explicitly way, the realization of a connected Lie group as an automorphism group follows from the subrigidity derived in $\S 3$.

This realization problem was first noticed by the author and also mentioned to the author by Peter Heinzner in September 2002 when I was visiting Ruhr-Universität, Bochum, Germany. Over years I was trapped in an effort to show every Grauert tube is Stein. Among other things, I am very grateful to Peter Heinzner for bringing my attention to $[\mathrm{H}-\mathrm{H}-\mathrm{K}]$ and $[\mathrm{D}-\mathrm{G}]$, which have helped settling down the Steiness part needed in this article, during my recent visit to Bochum in August 2004. I would also like to thank Jörg Winkelmann for many helpful discussions about this project during the Hayama Symposium 2002.

\section{Existence of Stein Grauert tubes}

Throughout this article, $(M, g)$ is assumed to be a connected real-analytic Riemannian manifold of dimension $\geq 2$. There exists a complex structure, the adapted complex structure, in a domain $\Omega(M) \subset T M$ which turns leaves of the Riemannian foliation on $\Omega(M) \backslash M$ into holomorphic curves. Let us denote by $\Omega(M)$ the maximal domain in which the adapted complex structure exists. With respect to this complex structure, the length square function $\rho(x, v):=|v|^{2}$ is strictly plurisubharmonic and satisfies the complex homogeneous Monge-Ampère equation $\left(d d^{c} \rho\right)^{n}=0$ on $\Omega(M) \backslash M$ with the initial condition $\left.\rho_{i} \bar{j}\right|_{M}=\frac{1}{2} g_{i j}$. The Grauert tube

$$
T^{r} M=\left\{(x, v): x \in M, v \in T_{x} M,|v|<r\right\}=\left\{\rho^{-1}\left[0, r^{2}\right)\right\}
$$

is the collection of tangent vectors of length less than $r$ equipped with the adapted complex structure; $M$ is the center and $r$ is the radius. In general the maximal domain $\Omega(M)$ is not a Grauert tube, $\Omega(M)$ is a Grauert tube if and only if $M$ is a symmetric 
space of rank one. There is a natural antiholomorphic involution $\sigma$ fixing every point of $M$,

$$
\sigma: \Omega(M) \rightarrow \Omega(M), \quad(x, v) \rightarrow(x,-v) .
$$

For two isometric real-analytic Riemannian manifolds $(M, g)$ and $(N, \kappa)$, the nature of the adapted complex structure will assure the biholomorphic equivalence of $\Omega(M)$ and $\Omega(N)$ and the biholomorphic equivalence of $T^{r} M$ and $T^{r} N$ as well. Given an isometry $h$ of $(M, g)$, the differential $d h$ acts as a biholomorphism on $\Omega(M)$;

$$
d h: \Omega(M) \rightarrow \Omega(M), \quad d h(x, v)=\left(h(x), h_{*} v\right) .
$$

The notation $F_{p}^{r}$ is reserved for the fiber passing $p \in M$,

$$
F_{p}^{r}:=\left\{(p, v): v \in T_{p} M,|v|<r\right\} .
$$

Since the complex structure we consider here is a local object, for each realanalytic Riemannian manifold, there exists an $r_{\max }(M) \geq 0$, the maximal radius such that the adapted complex structure can be well-defined on $T^{r_{\max }} M$. For compact, cocompact or homogeneous $M, r_{\max }(M)>0$. For any $r<r_{\max }(M)$, the complex manifold $T^{r} M \subset T^{r_{\max }} M$ with strictly pseudoconvex boundary defined by $\left\{\rho=r^{2}\right\}$.

It is shown in Theorem 5.2 of [Ka] that any Grauert tube over homogeneous space of radius $r<r_{\max }$ is complete hyperbolic. Another natural question to ask about is whether or not a Grauert tube is Stein.

Recall from [D-G] that an unramified Riemannian domain $D$ over a Stein manifold $X$ is a complex manifold $D$ together with a locally homeomorphic holomorphic map from $D$ to $X$ and that a pseudoconvex unramified Riemannian domain over a Stein manifold is itself Stein. It is clear that any pseudoconvex domain in a Stein manifold is Stein since we may take the imbedding as the holomorphic map.

We remark that a Grauert tube $T^{r} M$ is a Stein manifold for any $r \leq r_{\max }(M)$ provided $M$ is one of the following: compact; co-compact; homogeneous with nonnegative curvature. The first two cases are clear. Following the decomposition theorem (Theorem 7.1*, [C-G]) of Cheeger-Gromoll a homogeneous manifold $M$ of nonnegative curvature may be written as the product $\mathbb{R}^{k} \times M^{*}$ where $M^{*}$ is a compact homogeneous space of nonnegative curvature. Since the metric on $M$ is the product metric from $\mathbb{R}^{k}$ and $M^{*}$, it is clear that $r_{\max }(M)=r_{\max }\left(M^{*}\right)$ and $T^{r}(M) \subset \mathbb{C}^{k} \times T^{r_{\max }}\left(M^{*}\right)$ for all $r \leq r_{\max }$. For any $r \leq r_{\max }, T^{r}(M)$ has pseudoconvex boundary since the boundary is locally exhausted by the strictly plurisubharmonic function $-\log \left(r^{2}-\rho\right)$. Being a pseudoconvex domain in the Stein manifold $\mathbb{C}^{k} \times T^{r_{\max }}\left(M^{*}\right), T^{r_{\max }} M$ is Stein.

Though there is no example disproving the Steiness of any Grauert tube, the above three categories are the only complete classes that we are sure about the Steiness of any Grauert tubes over. For some very special kind of homogeneous spaces $M=G / K$, Halverscheid-Iannuzzi $[\mathrm{H}-\mathrm{I}]$ were able to construct a polar map from $T M$ to the Stein manifold $G_{\mathbb{C}} / i\left(K_{\mathbb{C}}\right)$. They show the maximal domain over a Heisenberg group 
is neither holomorphically separable nor holomorphically convex. We would like to remark that Grauert tubes constructed over their spaces are Stein. Their polar map is an unramified one therefore, Grauert tubes are pseudoconvex Riemann domains over the Stein manifold $G_{\mathbb{C}} / i\left(K_{\mathbb{C}}\right)$, they are Stein (cf. [D-G]).

A complex manifold $X$ is a complexification of $M$ if $M \subset X$ as a maximal totally real submanifold $\operatorname{dim}_{\mathbb{C}} X=\operatorname{dim}_{\mathbb{R}} M$. Utilizing his solution to the Levi problem, Grauert has proved there always exists a Stein complexification of a real-analytic manifold.

Though any two complexifications $\left(X_{1}, J_{1}\right)$ and $\left(X_{2}, J_{2}\right)$ of a real-analytic manifold $M$ are locally biholomorphic near $M$, i.e., there exist a neighborhood $U_{1}$ of $M$ in $X_{1}$ and a neighborhood $U_{2}$ of $M$ in $X_{2}$ such that $U_{1}$ and $U_{2}$ are biholomorphically equivalent, we still can not conclude there are some Stein Grauert tubes of small radii since it does not seem clear how to control the radius uniformly when the manifold is not relatively compact.

The following theorem, originally observed by Kutzschebauch in [Ku], states that a complexification can be chosen to be $G$-invariant if there is some kind of $G$-action on $M$.

Theorem $([\mathrm{H}-\mathrm{H}-\mathrm{K}])$. Let $X$ be a $G$-complexification of a real-analytic manifold $M$ where $G$ is a connected Lie group acting properly on $M$ as real-analytic diffeomorphisms. Then there exists a G-invariant Stein neighborhood of $M$ in X.

A group action $G$ on $M$ is proper if the mapping $G \times M \rightarrow M \times M,(g, m) \rightarrow$ $(\mathrm{gm}, \mathrm{m})$ is a proper map. A complexification $X$ of $M$ is called a $G$-complexification if the $G$-action on $M$ extends to a holomorphic $G$-action on $X$.

Though it is not clear whether a generic Grauert tube is Stein or not, a direct application of the above theorem would assert the existence of Stein Grauert tubes of small radii provided the centers have possessed some transitivity property. This would be enough for the purpose of solving the realization problem.

Proposition 2.1. Let $(M, g)$ be a homogeneous space. Then there exist $\varepsilon>0$ such that the Grauert tube $T^{\varepsilon} M$ is Stein and complete hyperbolic.

Proof. Let $G=\operatorname{Isom}_{0}(M)$, then $G$ acts properly on $M$ and acts holomorphically on the complexification $T^{r_{\max }} M$ of $M$. By [H-H-K], there exists a $G$-invariant Stein neighborhood $U$ of $M$ in $T^{r_{\max }} M$. For $p \in M$ we may assume the fiber $F_{p}^{\varepsilon}$, tangent vectors at $p$ of length less than $\varepsilon$, is contained in $U$. By the transitivity of the $G$-action, $G \cdot F_{p}^{\varepsilon}$ is the Grauert tube $T^{\varepsilon} M$. The Steiness follows since $T^{\varepsilon} M$ is now a strictly pseudoconvex domain in the Stein manifold $U$. 


\section{Subrigidity of domains}

Let Aut denote the automorphism group and Isom denote the isometry group; Aut 0 and Isom $_{0}$ denote the corresponding identity components. For any real-analytic Riemannian manifold $(M, g)$ such that $T^{r} M$ is not covered by the ball, the following rigidity results were proved in [Ka]: if $M$ is homogeneous then $\operatorname{Aut}\left(T^{r} M\right)=$ $\operatorname{Isom}(M)$.

We remark here that whenever the rigidity is mentioned, we always assume $\operatorname{dim} M>1$. When $\operatorname{dim} M=1$, a Grauert tube $T^{r} M$ is a Riemann surface. One essential feature of Grauert tubes is the symmetry on each fiber, each fiber is a disk bundle. In this section, we consider domains in a more general setting, perfect symmetry on fibers will not be asked for anymore. One crucial step in proving the rigidity is the Theorem $4.1 \mathrm{in}[\mathrm{Ka}]$ which has characterized the isometry group of $M$. We will show this kind of characterization still works and $\operatorname{Aut}(D)$ is dominated by $\operatorname{Isom}(M)$ provided there is some transitivity on $M$. Arguments will follow the spirits and methods developed in [Ka]. However, an extra piece of assumptions on the target Grauert tube is necessary; we assume Grauert tubes are Stein while the existence has been guaranteed in the last section.

Lemma 3.1. Let $D \subset T^{r} M$ be a connected domain containing $M$. If $f \in \operatorname{Aut}(D)$, $f(M)=M$, then $f=d u$ for some isometry u from $(M, g)$ to $(M, \kappa)$. Moreover, $f$ can be extended over the boundary $\partial D$.

Proof. From the construction of Grauert tubes, it is clear that $M$ is a maximal totally real submanifold of $D$. Let $\rho$ denote the length square function on $T^{r} M$, then $\rho \cdot f^{-1}$ is a strictly plurisubharmonic function on $D$. The Riemannian metric $g$ is induced from the Kähler form $i \partial \bar{\partial} \rho,\left.2 \rho_{i} \bar{j}\right|_{M}=g_{i j}$. Similarly, the Kähler form $i \partial \bar{\partial}\left(\rho \cdot f^{-1}\right)$ has induced a Riemannian metric $k$ on $M$. Let $u=\left.f\right|_{M}:(M, g) \rightarrow(M, \kappa)$. Then $u \in \operatorname{Isom}((M, g),(M, \kappa))$.

By the nature of the adapted complex structure, the differential $d u$ is a biholomorphic mapping from the Grauert tube $T^{r}(M, g)$ to the Grauert tube $T^{r}(M, \kappa)$, i.e. $d u \in \operatorname{Aut}\left(T^{r} M\right)$.

$d u^{-1} \cdot f: D \rightarrow T^{r} M$ is a holomorphic mapping. Since every complex manifold is locally Stein and $f=d u$ at the maximal totally real submanifold $M$, the identity principle implies that $f \equiv d u$ on $D$.

For $\left.(x, v) \in D, f(x, v)=d u(x, v)=\left(u(x), u_{*} v\right)\right)=\left(f(x), u_{*} v\right)$ is a bundle map acting fiberwise. It is clear that $f$ can be extended over the boundary of $D$.

Recall $\Omega(M)$ is the maximal domain in $T M$ such that the adapted complex structure is defined; $\sigma$ is the natural antiholomorphic involution in $\Omega(M)$ and the normsquare function $\rho$ is strictly plurisubharmonic in $\Omega(M)$. Sibony (Theorem 3, [S]) has asserted the hyperbolicity of any complex manifold equipped with a bounded strictly 
plurisubharmonic function. For domains in $\Omega(M)$ to be hyperbolic, the only thing we got to take care is the vertical direction.

One essential feature of Grauert tubes is that each fiber, which is diffeomorphic to a real ball, has perfect symmetry. We have tried to release this symmetry on the fiber to get some kind of rigidity result. However, it seems to us certain kind of transitivity on the domain is necessary for our purpose. We make the following definition.

Definition 3.2. A domain $D \subset \Omega(M)$ is $G$-homogeneous if

(1) there exists a connected subgroup $G \subset(\operatorname{Isom}(M) \cap \operatorname{Aut}(D))$ acting transitively on $M$;

(2) there exists a bounded open subset $F_{p} \subset T_{p} M$ such that $D=G \cdot F_{p}$.

With this extra piece of symmetry, Lemma 3.1 has the following refined form:

Proposition 3.3. Let $D$ be a $G$-homogeneous domain in $\Omega(M)$. If $f \in \operatorname{Aut}(D)$, $f(M)=M$, then $f=d u$ for some $u \in \operatorname{Isom}(M)$.

Proof. By Lemma 3.1, $f=d u$ for some isometry $u$ from the Riemannian manifold $(M, g)$ to the Riemannian manifold $(M, \kappa)$. By the assumption on $D$ there exist $G \subset \operatorname{Isom}(M), p \in M$ and $F_{p}$ such that $D=G \cdot F_{p}$.

The homogeneity will imply the equality of the two metrics $g$ and $\kappa$. Let $q=$ $u(p)=h(p)$ for some $h \in G$ and let $\left\{e_{1}, \ldots, e_{n}\right\}$ be an orthonormal basis of $F_{p}$ with respect to the metric $g$. Since $h$ is an isometry, $\left\{\left(e_{1}, \ldots, e_{n}\right) A\right\}$ is an orthonormal basis of $F_{q}$ with respect to the metric $g$ where $A \in O(n)$. On the other hand, $\left\{u_{*} e_{1}, \ldots, u_{*} e_{n}\right\}$ form an orthonormal basis of $F_{q}$ with respect to the metric $\kappa$, thus, there exists a $B \in \mathrm{GL}(n, \mathbb{R})$ such that

$$
\left(u_{*} e_{1}, \ldots, u_{*} e_{n}\right)=\left(e_{1}, \ldots, e_{n}\right) A B .
$$

Let us denote the matrix $A B=C=\left(C_{1}, \ldots, C_{n}\right)$ and $e=\left(e_{1}, \ldots, e_{n}\right)$ then

$$
\delta_{i j}=\left\langle u_{*} e_{i}, u_{*} e_{j}\right\rangle_{\kappa}=\left\langle e C_{i}, e C_{j}\right\rangle_{g}=C_{i} \cdot C_{j} .
$$

This shows $C$ is a matrix with orthonormal columns, thus $C \in O(n)$ and $B \in O(n)$ as well.

The orthonormal basis $\left\{u_{*} e_{1}, \ldots, u_{*} e_{n}\right\}$ of the metric $\kappa$ come from an orthogonal transformation of the orthonormal basis $\left\{e_{1}, \ldots, e_{n}\right\}$ of the metric $g$. Thus, $\kappa=g$ and $u \in \operatorname{Isom}(M, g)$.

Definition 3.4. A $\sigma$-invariant $G$-homogeneous domain $D \subset \Omega(M)$ is strictly pseudoconvex if every boundary point of $D$ away from $M$ is strictly pseudoconvex. 
A $G$-homogeneous domain is hyperbolic and hence its automorphism group is a Lie group. Let $D$ be a $\sigma$-invariant strictly pseudoconvex $G$-homogeneous domain in $\Omega(M)$ and let $\mathcal{L}$ denote the Lie algebra of $\operatorname{Aut}(D)$. Each $\xi \in \mathcal{L}$ may be written as

$$
2 \xi=\left(\xi+\left.\frac{d}{d t}\right|_{t=0}(\sigma \cdot(\exp t \xi) \cdot \sigma)\right)+\left(\xi-\left.\frac{d}{d t}\right|_{t=0}(\sigma \cdot(\exp t \xi) \cdot \sigma)\right):=\xi_{h}+\xi_{v} .
$$

Both $\xi_{h}$ and $\xi_{v}$ are in $\mathcal{L}$. It is clear that $\xi_{h}$ is tangent to $M$. We would like to show that $\xi_{v}$ is tangent to $M$ as well. Since $\xi_{v}$ is a left-invariant vector field on $\operatorname{Aut}(D)$, we have, for any $z \in D, g \in \operatorname{Aut}(D), g_{*}\left(\xi_{v}(z)\right)=\xi_{v}(g z)$ when viewing $\xi_{v}$ as the corresponding vector field on $D$.

For $x \in M$ we have $\xi_{v}(x)=2 i \operatorname{Im} \xi(x)$. Suppose there exists $p \in M$ such that $\xi_{v}(p) \neq 0$. Let $f_{0}=\exp t_{0} \xi_{v}$ for some $t_{0} \ll 1$. By the homogeneity of $M$ and the relation $\xi_{v}(g p)=g_{*}\left(\xi_{v}(p)\right),\left|\xi_{v}(x)\right|=$ constant for all $x \in M$ we have $M_{1}:=f_{0}(M)$ sitting on some level set $\{\rho=\delta\}$. Take the normal coordinate $U=\left\{x \in \mathbb{R}^{n}:|x|<\varepsilon\right\}$ around $p$ in $M$ so that the $(x, y)$ form a coordinate system of $\Omega(U)$. Since $U_{1}=: f_{0}(U) \subset\{\rho=\delta\}$, exp $t_{0} \xi_{v}(x)=$ $\left(x, y_{1}(x), y_{2}(x), \ldots, y_{n}(x)\right)$ with $\sum_{j}\left|y_{j}(x)\right|^{2}=\delta$. By the dimensional counting, every $y_{j}$ is a constant, i.e., $U_{1}=\left\{\left(x, \varepsilon_{1}, \varepsilon_{2}, \ldots, \varepsilon_{n}\right): x \in U\right\}, \sum_{j} \varepsilon_{j}^{2}=\delta$. There exists $\eta_{1} \in \mathscr{L}$ and $t_{1}>0$ so that $\exp t_{1} \eta_{1}(x, \varepsilon)=\exp t_{0}\left(-\xi_{v}\right)(x, \varepsilon)=x$ for all $(x, \varepsilon) \in U_{1}$. Then $\exp t_{1}\left(-\eta_{1}\right)\left(U_{1}\right)=\left\{\left(x, 2 \varepsilon_{1}, 2 \varepsilon_{2}, \ldots, 2 \varepsilon_{n}\right): x \in U\right\}=: U_{2}$ and $\exp t_{1}\left(-\eta_{1}\right) \cdot \exp t_{0} \xi_{v}(p) \in U_{2}$. Continuing the process, there exist $f_{j}:=$ $\exp t_{j}\left(-\eta_{j}\right) \ldots \exp t_{1}\left(-\eta_{1}\right) \cdot \exp t_{0} \xi_{v} \in \operatorname{Aut}(D), \lim _{j} f_{j}(p)$ is a strictly pseudoconvex boundary point, which forces $D$ to be a ball according to the generalized Wong-Rosay Theorem. Thus, $\xi_{v}=0$ for all $x \in M$ and $\xi$ is tangent to $M$ if $D$ is not the ball. We conclude

Proposition 3.5. Let $D$ be a $\sigma$-invariant strictly pseudoconvex $G$-homogeneous domain in $\Omega(M)$. If $D$ is not biholomorphic to the ball. Then $\operatorname{Aut}_{0}(D) \subset \operatorname{Isom}_{0}(M)$.

Following the arguments in $[\mathrm{Ka}]$, we are going to prove the subrigidity $\operatorname{Aut}(D) \subset$ $\operatorname{Isom}(M)$. For the rest of this section, we assume $D$ is a strictly pseudoconvex $\sigma$-invariant $G$-homogeneous domain in $\Omega(M), D$ is not biholomorphic to the ball. Proofs go exactly the same way as in $\S 7$ of $[\mathrm{Ka}]$; a brief explanation would be given in the following.

(I) First of all, a strictly pseudoconvex $G$-homogeneous domain in $\Omega(M)$ is complete hyperbolic since the boundary behavior is dominated by the strictly pseudoconvexity and the horizontal direction is determined by the transitivity of the $G$-action.

(II) Secondly, the following is clear from the $G$-homogeneity and Proposition 3.5:

$$
G \subset \operatorname{Aut}_{0}(D) \subset \operatorname{Isom}_{0}(M) .
$$

Given $f \in \operatorname{Aut}(D), D$ is also a $f \cdot G \cdot f^{-1}$-homogeneous domain centered at 
$N=f(M)$. By the homogeneity of the $G$-action,

$$
\begin{gathered}
N=f(M)=f(G \cdot p)=f\left(\operatorname{Aut}_{0}(D) \cdot p\right)=\operatorname{Aut}_{0}(D) \cdot f(p) ; \\
\left.\pi(N)=\pi \operatorname{Aut}_{0}(D) \cdot f(p)\right)=\operatorname{Aut}_{0}(D) \cdot \pi(f(p))=M .
\end{gathered}
$$

Thus, the argument in Lemma 7.1 of [Ka] goes through and $f(M) \cap T_{p} M \neq \varnothing$ for any $p \in M$.

(III) Since $D$ is complete hyperbolic and $G$-homogeneous, arguments in Proposition 7.2 of $[\mathrm{Ka}]$ can be transplanted here. Thus, the index of $G$ in $\operatorname{Aut}(D)$ is finite.

We conclude this section by the following theorem.

Theorem 3.6. Let $D$ be a $\sigma$-invariant strictly pseudoconvex G-homogeneous domain in $\Omega(M)$. Then either $\operatorname{Aut}(D) \subset \operatorname{Isom}(M)$ or $D$ is the ball.

Proof. As stated in (III), Aut $(D) / G=\left\{g_{j} G: g_{j} \in \operatorname{Aut}(D), j=1, \ldots, k\right\}$. Then $\psi(z)=\sum_{j=1}^{k} \rho\left(g_{j}(z)\right)$ is an $\pm \operatorname{Aut}(D)$-invariant strictly plurisubharmonic nonnegative function in $D$ where $\rho$ is the length square function in $D$. As $G$ acts transitively on $M$, the tangent space $T_{z}(D)$ can be decomposed as, for any $z \in D$,

$$
T_{z}(D)=T_{z}(G \cdot z)+T_{z}\left(T_{\pi(z)} M \cap D\right)
$$

where $\pi$ is the fiber projection $\pi(x, v)=x$ for all $v \in T_{x} M$.

Since $\psi$ is constant in $G \cdot z$, every critical point of the function $f_{z}:=\left.\psi\right|_{T_{\pi(z)} M \cap D}$ is a critical point of $\psi$ and every critical point of $\psi$ occurs at the critical points of the functions $f_{z}$.

As $\psi$ is strictly plurisubharmonic, the above decomposition implies that the real Hessian of $f_{z}$ is positive definite on the tangent space $T_{z}\left(T_{\pi(z)} M \cap D\right)$. Since $f_{z}$ is proper on the fiber, it follows that there is exactly one critical point of $f_{z}$ which turns out to the minimal point. Since $\psi \cdot \sigma=\psi$, the minimum of $f_{z}$ occurs at $\pi(z)$. That is to say that the set of critical points of $\psi$ is exactly $M$.

Let $f \in \operatorname{Aut}(D), f(M)=N$, then $N$ is the critical point set of $\psi$ since $\psi$ is Aut $(D)$-invariant. We conclude that $N=M$ and $f \in \operatorname{Isom}(M)$ following from Proposition 3.3. The inclusion $\operatorname{Aut}(D) \subset \operatorname{Isom}(M)$ is concluded.

\section{Realizing a connected Lie group as an automorphism group}

Let $G$ be a connected real Lie group with Lie algebra $g$. Given a positive definite inner product $\langle$,$\rangle on T_{e} G=g$, we may endow $G$ with the associated left invariant Riemannian metric $g$. Every Lie group is real-analytic, since it is locally diffeomorphic to the Lie algebra $g$ through the exponential map. Thus $(G, g)$ is a real-analytic Riemannian manifold. Furthermore, $(G, g)$ is a homogeneous space with trivial tangent bundle $G \times T_{e} G$. 
It is clear that the left translation group $L(G)$ is a subgroup of $\operatorname{Isom}(G, g)$ and $L(G)$ acts transitively on $G$. Furthermore, the transitivity implies the diffeomorphic equivalence of $G$ with $\operatorname{Isom}(G, g) / K$ and with $\operatorname{Isom}_{0}(G, g) / K_{0}$ where

$$
K=\{h \in \operatorname{Isom}(G, g): h \cdot e=e\}
$$

is the isotropy group at $e \in G$ and $K_{0}=K \cap \operatorname{Isom}_{0}(G, g)$. The following two equations are immediate.

$$
\operatorname{Isom}(G, g) \simeq L(G) \cdot K, \quad \operatorname{Isom}_{0}(G, g) \simeq L(G) \cdot K_{0} .
$$

From now on, we denote the homogeneous Riemannian manifold $(G, g)$ as $(M, g)$ where $G$ is a connected Lie group of dimension $n \geq 2$ and $g$ is a left invariant real-analytic metric on $G$. We would like to construct a $G$-homogeneous strictly pseudoconvex domain and then further perturb the domain to eliminate additional automorphisms such that the automorphism group of the resultant domain is $G$. We emphasize that our methods work for both compact and noncompact Lie groups as long as the Lie group is connected. The dimensional condition $n \geq 2$ has to be added here since the main idea we used here follows from the rigidity argument of Grauert tubes while the rigidity of Grauert tubes fails when the center is of dimension one.

Let $(M, g)=(G, g)$ be as above. Since it is homogeneous, there exist $\delta>0$ such that the Grauert tube $T^{\delta} M$ is Stein and the Grauert tube $T^{2 \delta} M$ still exists. Let $\rho$ denote the length square function; $\sigma$ denote the natural antiholomorphic map in $T^{2 \delta} M$ and $F_{p}^{r}$ denote tangent vectors at $p \in M$ of length less than $r, F_{p}^{r}:=\{(p, v)$ : $\left.v \in T_{p} M,|v|<r\right\}$.

Let $\left\{e_{1}, \ldots, e_{n}\right\}$ be an orthonormal basis - with respect to the metric $g-$ of $F_{p}^{2 \delta}$, $K$ be the isotropy group of $(M, g)$ at $p \in M$. Then $K \subset O(n)$ when we view $F_{p}^{2 \delta}$ as a subspace of the real vector space generated by the orthonormal basis $\left\{e_{1}, \ldots, e_{n}\right\}$.

Let $a_{j}=\delta e_{j} \in F_{p}^{2 \delta}, j=1, \ldots, n$. Choose a ball $B_{\varepsilon} \subset F_{p}^{2 \delta}$ centered at $a_{1}$ of radius $\varepsilon \ll \delta$ and a family of orthogonal transformations $f_{j} \in O(n)$ such that $f_{j}\left(a_{1}\right)=a_{j}, j=2, \ldots, n$.

Let $\eta_{1}(x)=\left(x-a_{1}\right)^{4 l}, l \geq 1$, be a real-analytic function on $B_{\varepsilon}$. Convoluting with some cut-off function, we may assume $\eta_{1}$ has compact support $C_{1} \subset B_{\varepsilon}$.

Denoting $f_{1}=\mathrm{id}$, we define a function $\eta_{2}$ on $F_{p}^{2 \delta}$ as follows:

$$
\eta_{2}\left(f_{j}(x)\right)=\eta_{2}\left(\sigma\left(f_{j}(x)\right)\right)=\frac{1}{j} \eta_{1}(x) \text { for all } x \in F_{p}^{2 \delta}, j=1, \ldots, n \text {. }
$$

The ball $B_{\varepsilon}$ can be arranged so small that $\left\{\sigma^{i}\left(f_{j}\left(B_{\varepsilon}\right)\right): i=0,1 ; j=1, \ldots, n\right\}$ are pairwise disjoint. It is clear that $\eta_{2}$ is a $\sigma$-invariant function in $F_{p}^{2 \delta}$ with compact support $C_{2}$ contained in $\bigcup_{j=1}^{n}\left(f_{j}\left(B_{\varepsilon}\right) \cup \sigma\left(f_{j}\left(B_{\varepsilon}\right)\right)\right)$.

Since the tangent bundle of a Lie group is trivial, $T M=G \times T_{p} M$. We define a real-analytic functions $\eta$ in $T^{2 \delta} M=G \times F_{p}^{2 \delta}$ by setting

$$
\eta(h \cdot x)=\eta_{2}(x) \text { for all } h \in G, x \in F_{p}^{2 \delta} .
$$


Clearly, $\eta$ is a nonnegative $G$-invariant and $\sigma$-invariant real-analytic function on $T^{2 \delta} M$. Recall $\rho$ is the length square function of the tangent vectors which is $G$-invariant and strictly plurisubharmonic and $T^{\delta} M=\left\{\rho^{-1}\left(\left[0, \delta^{2}\right)\right)\right\}$. Define the function, for some $0<\varepsilon^{\prime} \ll 1$,

$$
\hat{\rho}=\rho-\delta^{2}+\varepsilon^{\prime} \eta
$$

Shrinking $\varepsilon^{\prime}$ if necessary such that all the 0 -th, first and second order derivatives of $\varepsilon^{\prime} \eta$ are well under control. Thus, we may assume the function $\hat{\rho}$ is strictly plurisubharmonic. It is $G$ and $\sigma$ invariant since both $\rho$ and $\eta$ are.

Let $D$ be a domain defined by the function $\hat{\rho}$,

$$
D:=\left\{z \in T^{2 \delta} M: \hat{\rho}(z)<0\right\} .
$$

It is clear that $D$ is a $\sigma$-invariant strictly pseudoconvex $G$-homogeneous domain in $T^{\delta} M$. In order to apply Theorem 3.6 to conclude the subrigidity, we need to show the domain $D$ is not biholomorphic to the ball. Some background on Chern-Moser normal form is needed here.

In the fundamental paper [C-M], Chern and Moser have associated to every strictly pseudoconvex point $p$ in a hypersurface $H$ a family of local invariants, namely a neighborhood $U_{p}$ of $p$ in $H$ is biholomorphically equivalent to a neighborhood $V_{q}$ of $q$ in a hypersurface $S$ if and only if the associated families of invariants at $p$ and at $q$ are the same. These invariants are given by the coefficients of certain normal form of the defining function which we briefly explain in the following. Let $\psi$ be a local defining function of the $(2 n-1)$-dimensional hypersurface $H$ near the point $p$, say inside a coordinate chart. Since $p$ is strictly pseudoconvex the Levi form is positive definite and the defining function can be transformed through some linear translation and proper holomorphic transformations to the following:

$$
v=|z|^{2}+F(z, \bar{z}, w)
$$

where $z=\left(z_{1}, \ldots, z_{n-1}\right) \in \mathbb{C}^{n-1}, w=u+i v \in \mathbb{C}$. Using the transformations $z^{*}=z+f(z, w), w^{*}=w+g(z, w)$ Chern and Moser have simplified (4.7) to

$$
v^{*}=\left|z^{*}\right|^{2}+N_{24}\left(u^{*}\right) z^{* 2} \bar{z}^{* 4}+N_{42}\left(u^{*}\right) z^{* 4} \bar{z}^{* 2}+\sum_{j, k \geq 2, j+k \geq 7} N_{j k}\left(u^{*}\right) z^{* j} \bar{z}^{* k}
$$

Furthermore this transformation, and hence all of the coefficients $N_{j k}$, is made unique when certain normalizations on $f$ and $g$ are made.

For the hyperquadric $Q=\left\{v=|z|^{2}\right\}$, the unbounded model of the sphere, all invariants $N_{j k}$ vanish.

Lemma 4.1. $D$ is not biholomorphic to the ball. 
Proof. It is standard that given any $n$-dimensional totally real closed submanifold $X \subset B^{n}$ there exists $\varphi \in \operatorname{Aut}\left(B^{n}\right)$ such that $\varphi(X)=\mathbb{R}^{n} \cap B^{n}$.

Suppose there exists a biholomorphic map $f: D \rightarrow B^{n}$, then $f(M)$ is a maximal totally real closed submanifold of $B^{n}$ since $M$ is such kind of submanifold of $D$. Without loss of generality, we may assume $f(M)=\mathbb{R}^{n} \cap B^{n}$. Adopting the argument used in the proof of Lemma 3.1, we see $f$ is a bundle map, $f=d u$ for some isometry $u$ from $(M, g)$ to $\left(\mathbb{R}^{n} \cap B^{n}, g^{*}\right)$.

Thus, the biholomorphic map $f$ can be extended holomorphically over the boundary $\partial D$ and thus the Chern-Moser normal form of $\hat{\rho}$ at any boundary point of $D$ has the local expression $v=|z|^{2}$.

However, the defining function near $a_{1} \in \partial D$ and the defining function near generic boundary points differ by some $4 l$-order terms. In the construction of the normal form, we see the orders will not decrease. Thus the Chern-Moser normal form at $a_{1}$ will not be the same as the Chern-Moser normal form at generic boundary points which we assume to be $v=|z|^{2}$. A contradiction.

It is clear $G$ is contained in the automorphism group of $D$ since $D$ is $G$-invariant. Theorem 3.6 along with (4.2) implies

$$
L(G)<\operatorname{Aut}(D)<\operatorname{Isom}(M) \simeq L(G) \cdot K .
$$

The last step is to eliminate those automorphisms coming form the isotropy group.

Theorem 4.2. $D$ is a complete hyperbolic Stein manifold with $\operatorname{Aut}(D)=G$.

Proof. $D$ is complete hyperbolic since it is $G$-homogeneous with strictly pseudoconvex boundary. It is Stein since $D$ is a strictly pseudoconvex domain in the Stein manifold $T^{\delta} M$.

It remains to show if $h \in K \cap \operatorname{Aut}(D)$, then $h$ is the identity map. By the construction of $\eta_{2}$ at (4.3) and hence the construction of the defining function $\hat{\rho}$, we see that there exist neighborhoods $U_{j}$ of $a_{j}$ on the hypersurface $\partial D \cap F_{p}^{\delta}$ such that points in $U_{i}-a_{i}$ all have norms different from norms of points in $U_{j}-a_{j}$ for $i \neq j$. Besides, every point in $U_{i}-a_{i}$ has norm $<\delta$ and $\left|a_{i}\right|=\delta, i=1, \ldots, n$.

Since $K$ is a subgroup of $\operatorname{Isom}(M), h$ is a linear and norm-preserving isomorphism of $F_{p}^{\delta} \cap D$. In fact, $h$ is the restriction of an orthogonal transformation in $F_{p}^{\delta}$. Thus, $h$ is a norm preserving map from $\partial D \cap F_{p}^{\delta}$ to $\partial D \cap F_{p}^{\delta}$ and the only possibility for $h$ is either $h\left(a_{j}\right)=a_{j}$ or $h\left(a_{j}\right)=\sigma\left(a_{j}\right)=-a_{j}, j=1, \ldots, n$.

Recall that $a_{j}=\delta e_{j}$, the linearity of $h$ implies either $h\left(e_{j}\right)=e_{j}$ or $h\left(e_{j}\right)=-e_{j}$ for all $j$. Thus, $h$ is either the identity map or the negative identity, in other words, $h=\mathrm{id}$ or $h=\sigma$. The second case is not possible since then $h$ is antiholomorphic rather than holomorphic. We conclude $h=\mathrm{id}, K=\mathrm{id}$ and $\operatorname{Aut}(D)=L(G) \simeq G$. 
Vol. 82 (2007)

\section{References}

[B-D] E. Bedford and J. Dadok, Bounded domains with prescribed group of automorphisms. Comment. Math. Helv. 62 (1987), 561-572. Zbl 0647.32027 MR 0920057

[C-G] C. Cheeger and D. Gromoll, On the structure of complete manifolds of nonnegative curvature. Ann. of Math. 96 (1972), 413-443. Zbl 0246.53049 MR 0309010

[C-M] S.-S. Chern and J. Moser, Real hypersurfaces in complex manifolds. Acta Math. 133 (1974), 219-271. Zbl 0302.32015 MR 0709144

[D-G] F. Docquier and H. Grauert, Levisches Problem und Rungescher Satz für Teilgebiete Steinscher Mannigfaltigkeiten. Math. Ann. 140 (1960), 94-123. Zbl 0095.28004 MR 0148939

[H-I] S. Halverscheid and A. Iannuzzi, Maximal complexifications of certain homogeneous Riemannian manifolds. Trans. Amer. Math. Soc. 355 (11) (2003), 4581-4594. Zbl 1039.32009 MR 1990763

[H-H-K] P. Heinzner, A. Huckleberry and F. Kutzschebauch, A real analytic version of Abels' theorem and complexifications of proper Lie group actions. In Complex analysis and geometry (Trento, 1993), Lecture Notes in Pure and Appl. Math. 173, Dekker, New York 1996, 229-273. Zbl 0861.32011 MR 1365977

[Ka] S.-J. Kan, On rigidity of Grauert tubes over homogeneous Riemannian manifolds. J. Reine Angew. Math. 577 (2004), 213-233. Zbl 1069.53040 MR 2108219

$[\mathrm{Ku}] \quad$ F. Kutzschebauch, Eigentliche Wirkungen von Liegruppen auf reell-analytischen Mannigfaltigkeiten. Schriftenreihe des Graduiertenkollegs Geometrische und Mathematische Physik 5, Ruhr-Universität Bochum, Germany, 1994. Zbl 0840.22017

[S] N. Sibony, A class of hyperbolic manifolds. In Recent developments in several complex variables (Princeton University, 1979), Ann. of Math. Stud. 100, Princeton University Press, Princeton, N.J., 1981, 357-372. Zbl 0476.32033 MR 0627768

[S-Z] R. Saerens and W. Zame, The isometry groups of manifold and the automorphism groups of domains. Trans. Amer. Math. Soc. 301 (1) (1987), 413-429. Zbl 0621.32025 MR 0879582

[W] J. Winkelmann, Realizing connected Lie groups as automorphism groups of complex manifolds. Comment. Math. Helv. 79 (2) (2004), 285-299. Zbl 1056.32022 MR 2059433

Received December 14, 2004; revised August 4, 2005

Su-Jen Kan, Institute of Mathematics, Academia Sinica, Taipei, Taiwan

E-mail:kan@math.sinica.edu.tw 